\title{
Erratum: Predicting phonon dispersion relations and lifetimes from the spectral energy density [Phys. Rev. B 81, 081411(R) (2010)]
}

\author{
John A. Thomas, Joseph E. Turney, Ryan M. Iutzi, Cristina H. Amon, and Alan J. H. McGaughey \\ (Received 17 June 2015; published 30 June 2015) \\ DOI: 10.1103/PhysRevB.91.239905 \\ PACS number(s): 63.20.-e, 63.22.-m, 66.70.-f, 99.10.Cd
}

Feng et al. recently proved that Eq. (4) in the original paper represents the phonon spectral energy density [1]. We agree with their finding and believe that Eq. (4) can be used to extract phonon frequencies and lifetimes from molecular dynamics simulations. The previous Erratum on this paper [2] was the result of an error in a computer code different than the one used to generate the data for the original paper. Fixing the code led us to the same conclusion as Feng et al. As such, we believe that all the results in the original paper are reliable.

As noted by Feng et al., care should be taken for phonon modes with the same wave vector and similar frequencies, where fitting separate Lorentzian functions to the individual peaks in the spectral energy density may be challenging. In such cases, the normal mode decomposition technique, which performs the analysis at the mode level as opposed to the wave vector level, may be required.

[1] T. Feng, B. Qiu, and X. Ruan, J. Appl. Phys. 117, 195102 (2015).

[2] J. A. Thomas, J. E. Turney, R. M. Iutzi, C. H. Amon, and A. J. H. McGaughey, Phys. Rev. B 90, 039901(E) (2014). 